\title{
Correction to: Deep learning early warning system for embryo culture conditions and embryologist performance in the ART laboratory
}

\author{
Charles L. Bormann ${ }^{1} \cdot$ Carol Lynn Curchoe ${ }^{2}$ (D) Prudhvi Thirumalaraju ${ }^{3} \cdot$ Manoj K. Kanakasabapathy $^{3}$. \\ Raghav Gupta ${ }^{3} \cdot$ Rohan Pooniwala $^{3} \cdot$ Hemanth Kandula ${ }^{3} \cdot$ Irene Souter $^{1} \cdot$ Irene Dimitriadis $^{1} \cdot$ Hadi Shafiee $^{3}$
}

Published online: 14 May 2021

(C) Springer Science+Business Media, LLC, part of Springer Nature 2021

\section{Correction to: Journal of Assisted Reproduction and} Genetics

https://doi.org/10.1007/s10815-021-02198-x

The original article unfortunately contained an error. The author, Charles L. Bormann, should be listed as the first author in the article.

The original article has been corrected.

Publisher's note Springer Nature remains neutral with regard to jurisdictional claims in published maps and institutional affiliations.

The online version of the original article can be found at https://doi.org/ 10.1007/s10815-021-02198-x

Carol Lynn Curchoe

cburton@fertilitylabsciences.com

1 Massachusetts General Hospital Fertility Center, Obstetrics/ Gynecology/Reproductive Endocrinology and Infertility, Boston, MA, USA

2 Colorado Center for Reproductive Medicine, Newport Beach, CA, USA

3 Division of Engineering in Medicine, Department of Medicine, Brigham and Women's Hospital, Harvard Medical School, Boston, MA, USA 